\title{
Evaluation of Whole Saliva Antioxidant Capacity in Patients with Periodontal Diseases
}

\author{
Arash Azizi' ${ }^{1}$, Fatemeh Sarlati ${ }^{2}$, Azam Parchakani ${ }^{3}$, Somayeh Alirezaei ${ }^{{ }^{*}}$ \\ ${ }^{1}$ Oral and Maxillofacial Medicine Department, Islamic Azad University, Tehran, Iran \\ ${ }^{2}$ Islamic Azad University Dental Branch of Tehran, Tehran, Iran \\ ${ }^{3}$ DMD (Doctor of Dental Medicine), Tehran, Iran \\ Email: ${ }^{\text {dr.somayehalirezaei@gmail.com }}$
}

Received 7 March 2014; revised 9 April 2014; accepted 17 April 2014

Copyright (C) 2014 by authors and Scientific Research Publishing Inc.

This work is licensed under the Creative Commons Attribution International License (CC BY). http://creativecommons.org/licenses/by/4.0/

(c) (i) Open Access

\begin{abstract}
Background and purpose: Periodontitis is the inflammation of the supporting tissues of teeth. In this disease, overproduction of free radicals by polymorphonuclears (PMNs) will result in the imbalance between saliva's oxidants and antioxidants. Therefore, due to insufficient levels of antioxidants, free radicals can start chain reactions that eventually will lead to destruction of periodontal tissues. The purpose of this research is to compare the amount of whole saliva's antioxidant capacity in people with major periodontitis with that of healthy people. Methods and materials: In this case-control study, the amount of antioxidants capacity and saliva antioxidants were measured in 43 cases with periodontitis, as well as in 43 healthy people (controls); and the measurements were compared between the two groups. The amount of antioxidants capacity and their saliva antioxidants capacity was measured by FRAP (ferric reducing ability of plasma) and with the help of a Spectrophotometer. Findings: Average and Standard deviation of saliva's antioxidants was 184.25 \pm 63.209 in subject group and $399.419 \pm 90.298$ micro moles in control group. Using the T-TEST revealed that the difference in capacity of whole saliva's antioxidants in two groups is significant. Conclusion: whole saliva's antioxidants capacity is less among patients with periodontitis compared to control group.
\end{abstract}

\section{Keywords}

Anti-Oxidant, Chronic Periodontitis, Saliva

\footnotetext{
${ }^{*}$ Corresponding author.
}

How to cite this paper: Azizi, A., Sarlati, F., Parchakani, A. and Alirezaei, S. (2014) Evaluation of Whole Saliva Antioxidant Capacity in Patients with Periodontal Diseases. Open Journal of Stomatology, 4, 228-231. 


\section{Introduction}

Periodontitis is an inflammatory disease of supporting tissues of teeth caused by specific microorganisms. Activation of immune system may lead to progressive destruction of the periodontal ligament and alveolar bone including pocket formation or gingival recession or both. Bacterial products can also affect the immune system that may result in tissue damage if left untreated [1]. While reactive oxygen species (ROS) or free radicals such as $\left(\mathrm{O}_{2}\right)$ and $\mathrm{H}_{2} \mathrm{O}_{2}$ release from PMNs during phagocytosiscan eliminating periodontal pathogens, they may also damage the host cells.

ROS are extremely toxic and can result in DNA LPO (lipid peroxidation), protein damage, enzyme oxidation and stimulation of monocytes and macrophages to release pre-inflammatory cytokines [1] [2], which in turn may cause oxidative stress and disruption of cell integrity [3]. Oxidative stresses could also damage connective tissues and cause bone lysis around the tooth root resulting in loosening the tooth [4].

Antioxidants are released by PMNs, and they protect the body against reactive oxygen species. Antioxidants also exist in submandibular and parotid salivary glands as a normal anti-bacterial component [5]. Antioxidant alterations in some inflammatory and pre-cancerous diseases have been assessed and confirmed [6].

While some studies have shown that antioxidants levels are changedin periodontitis [7]-[10], some other ones have failed to show this [9]. Considering contrary results of previous studies on this issue, this study was conducted to assess the level of whole saliva antioxidants patients with chronic periodontitis.

\section{Methods and Materials}

This case control analytical research which was performed on 43 patients who presented to the oral and maxillofacial medicine department, and 43 healthy controls. Exclusion criteria were as follows: 1) taking regular medication and vitamins in the previous month; 2) history of heavy cigarette smoking in the last two years; 3) history of periodontal treatment in the last two years; 4) pregnancy. Written consent was taken from all patients and controls. After performing phase 1 of periodontal treatment (scaling and oral hygiene) 3 mL of non-stimulated saliva was collected during 5 minutes between 10 - 12 a.m. Tubes were kept in the dry ice and were sent to ErfanLabratoary with a specific code. Samples were kept in 4 degrees Celsius for 10 minutes, and then were centrifuged at $4000 \mathrm{rpm}$ in minus 80 degrees Celsius. All samples were collected in the same day (same temperature and humidity) and the level of antioxidants were calculated by FRAP method (ferric reducing ability of plasma) with the use of pharmacia spectrophotometer, and ENGLAN ultropec 3000 biotech (made in McKinley Scientific). Whole plasma antioxidants were evaluated by means of Benzie et al. method [10], which is based on plasma and saliva capacity to reduce $\mathrm{Fe}^{+3}$ to $\mathrm{Fe}^{+2}$ in the presence of TPTZ (2,4,6,-tripyridyl-s-triazine). Fe ${ }^{+3}-\mathrm{TPTZ}_{\text {com- }}$ plex is reduced under acidic conditions by a reducing agent to form $\mathrm{Fe}^{+2}-\mathrm{TPTZ}$. The production of Fe $\mathrm{F}^{+2}-\mathrm{TPTZ}^{\mathrm{T}}$ easily detectable and measurable because of its intense blue color with a maximum absorption at wavelength $593 \mathrm{~nm}$. Therefore, the determining factor to evaluate the speed of $\mathrm{Fe}^{2}$-TPTZ complex formation and production of blue color is the reducing ability of samples. As a result, this method is able to determine every anti-oxidant component which has a reductive potential. All sample solutions for measuring whole saliva antioxidants were gathered in the same day, and all utensils were washed by $10 \%$ Nitric acid and distilled water. Then the samples were kept in minus 80 degrees Celsius until the test date. Acetate buffers were prepared by mixing 3.1 grams of sodium acetate in $16 \mathrm{~mL}$ of acetic acid. Then, by adding distilled water the volume was increased to 1 liter; and the solution's PH was set to 3.6. The main reactive solution was a mixture of $2.5 \mathrm{~mL}$ of TPTZ, $2.5 \mathrm{~mL}$ of iron chloride plus $25 \mathrm{~mL}$ of acetate buffer. During the test, temperature was set to 37 degrees Celsius. Spectrophotometer at 593 nanometer wavelength converted to zero by pouring 900 microliter of solution into the cuvette. The reaction started by adding 30 microliter of gathered saliva that was earlier centrifuged at 4 degree Celsius. Absorption was measured at the beginning of the reaction and after 8 minutes. The differences between final and initial absorptions were measured and determined using the standard curve, which were drawn by using iron sulfate. The obtained data from regression equation was a base for the statistical analyses, which was done using the SPSS ver: 16 software and T-TEST.

\section{Findings}

The mean age of case group was $48.3 \pm 10.9$ years, and that of control group was $38 \pm 7.27$ years.

The results show that the average and standard deviation capacity of whole saliva antioxidants was $184.256 \pm$ 63.209 micro moles per $\mathrm{mL}$ in case group. By using T-TEST, the decrease in capacity of whole saliva antioxidant in case group was found to be statistically significant (Table 1). 
Table 1. Total antioxidant concentration in case and control groups.

\begin{tabular}{ccccccc}
\hline variables & number & Maximum & Minimum & Mean & SD & P-value \\
\hline TAO in case group & 43 & 196 & 516 & 399.42 & 90.3 & P $<0.001$ \\
TAO in control group & 43 & 96 & 336 & 184.26 & 63.21 & \\
\hline
\end{tabular}

\section{Discussion}

Our study showed that the amount of antioxidant in saliva of patients was lower than that of healthy control group. The results of this research were similar to that of Miricesue et al. study. That study showed that the amount of whole saliva's antioxidants in smokers, patients with lichen planus and periodontitis were less than healthy subjects [11]. In addition, in Novakovic et al. study, the reduction of antioxidant was also seen and confirmed [12].

In 2010, Kim al found out that the whole saliva antioxidants level increases after scaling [2].

In contrast, Moore et al. showed that there was no difference in amount and activity of antioxidants in case and healthy control groups, however, the number of patients in that study was not enough, and urea acid was considered as the most important antioxidant of saliva [13].

Chapple et al. study also showed that the amount of antioxidants is similar in patients with mild or severe periodontitis and healthy people. But in gingival crevicular fluid (GCF), the amount of antioxidants in case group was less than that of normal subjects. In addition, after scaling the amount of total antioxidant capacity (TAOC) in case group raised to the normal level [14].

Pathogens like $P$. gingivalis also secret their own antioxidants which enable them to resist against the killing effect of free radicals of neutrophils [15].

In saliva of healthy people, there is a balance between oxidants and antioxidants, while in that of unhealthy individuals, oxidants have a higher level that can cause oxidative stresses [4].

In periodontitis, the antioxidant system including superoxide dismutase (SOD), uric acid, ascorbic acid, Keto ferrule, myeloperoxidase, glutathione and albumin will be suppressed. SOD is one of the most important antioxidants that catalyze ions, and it protects the cells against the harmful effects of ROS. SOD also decreases significantly in the deep periodontal pocket [2].

A recent study has shown the importance of defensive role of antioxidants in saliva.

In conclusion, regarding the huge difference in the level of antioxidants between healthy individuals and patients, it is fair to conclude that production of ROS has the principal role in the initiation of the periodontal disease. Early diagnosis of periodontal disease is the best way to control the disease.

More researches are needed to further clarify the results of this study.

\section{Conclusion}

According to the results of this study, the average amount of antioxidants in case group is significantly lower than that of healthy group, which shows the necessity of early diagnosis and treatment of periodontitis.

\section{Acknowledgments}

Hereby, we are thankful to those who helped us in this article including Erfan's laboratory and medical research center of Azad University.

\section{References}

[1] Loesche, W.J. and Grossman, N.S. (2001) Periodontal Disease as a Specific, Albeit Chronic, Infection: Diagnosis and Treatment. Clinical Microbiology Reviews, 14, 727-752. http://dx.doi.org/10.1128/CMR.14.4.727-752.2001

[2] Kim, S.C., Kim, O.S., Kim, O.J., Kim, Y.J. and Chung, H.J. (2010) Antioxidant Profile of Whole Saliva after Scaling and Root Planning in Periodontal Disease. Journal of Periodontal \& Implant Science, 40, 164-171. http://dx.doi.org/10.5051/jpis.2010.40.4.164

[3] Diab-Ladki, R., Pellat, B. and Chahine, R. (2003) Decrease in the Total Antioxidant Activity of Saliva in Patients with Periodontal Diseases. Clinical Oral Investigations, 7, 103-107. http://dx.doi.org/10.1007/s00784-003-0208-5 
[4] Wei, D., Zhang, X.L., Wang, Y.Z., Yang, C.X. and Chen, G. (2010) Lipid Peroxidation Levels, Total Oxidant Status and Superoxide Dismutase in Serum, Saliva and Gingival Crevicular Fluid in Chronic Periodontitis Patients before and after Periodontal Therapy. Australian Dental Journal, 55, 70-78. http://dx.doi.org/10.1111/j.1834-7819.2009.01123.x

[5] Nikolaev, I.V., Kolobkova, L.N., Landesman, E.O., Stepanov, E.V. and Koroleva, O.V. (2008) Antioxidant and Peroxidase Activity of Saliva in Patients with Inflammatory Periodontal Diseases and Ability of Their Correction. Biomed Khim, 54, 454-462.

[6] Azizi, A. and Farshchi, F. (2012) Comparison of Salivary and Plasma Antioxidant Levels in Lichen Planus Patients and Healthy Subjects. Journal of Oral Pathology \& Medicine, 41, 524-526.

[7] Akalin, F.A., Baltacioğlu, E., Alver, A. and Karabulut, E. (2007) Lipid Peroxidation Levels and Total Oxidant Status in Serum, Saliva and Gingival Crevicular Fluid in Patients with Chronic Periodontitis. Journal of Clinical Periodontology, 34, 558-565. http://dx.doi.org/10.1111/j.1600-051X.2007.01091.X

[8] Baltacioğlu, E., Akalin, F.A., Alver, A., Balaban, F., Unsal, M. and Karabulut, E. (2006) Total Antioxidant Capacity and Superoxide Dismutase Activity Levels in Serum and Gingival Crevicular Fluid in Post-Menopausal Women with Chronic Periodontitis. Journal of Clinical Periodontology, 33, 385-392. http://dx.doi.org/10.1111/j.1600-051X.2006.00923.x

[9] Panjamurthy, K., Manoharan, S. and Ramachandran, C.R. (2005) Lipid Peroxidation and Antioxidant Status in Patients with Periodontitis. Cellular \& Molecular Biology Letters, 10, 255-264.

[10] Sculley, D.V. and Langley-Evans, S.C. (2003) Periodontal Disease Is Associated with Lower Antioxidant Capacity in Whole Saliva and Evidence of Increased Protein Oxidation. Clinical Science, 105, 167-172. http://dx.doi.org/10.1042/CS20030031

[11] Valko, M., Leibfritz, D., Moncol, J., Cronin, M.T., Mazur, M. and Telser, J. (2007) Free Radicals and Antioxidants in Normal Physiological Functions and Human Disease. The International Journal of Biochemistry \& Cell Biology, 39, 44-84. http://dx.doi.org/10.1016/j.biocel.2006.07.001

[12] Benzie, I.F. and Strain, J.J. (1996) The Ferric Reducing Ability of Plasma (FRAP) as a Measure of "Antioxidant Power”: The FRAP Assay. Analytical Biochemistry, 239, 70-76. http://dx.doi.org/10.1006/abio.1996.0292

[13] Moore, S., Calder, K.A., Miller, N.J. and Rice-Evans, C.A. (1994) Antioxidant Activity of Saliva and Periodontal Disease. Free Radical Research, 21, 417-425. http://dx.doi.org/10.3109/10715769409056594

[14] Chapple, I.L., Mason, G.I., Garner, I., Matthews, J.B., Thorpe, G.H., Maxwell, S.R. and Whitehead, T.P. (1997) Enhanced Chemiluminescent Assay for Measuring the Total Antioxidant Capacity of Serum, Saliva and Crevicular Fluid. Annals of Clinical Biochemistry, 34, 412-421. http://dx.doi.org/10.1177/000456329703400413

[15] Miricescu, D., Greabu, M., Totan, A., Didilescu, A. and Radulescu, R. (2011) The Antioxidant Potential of Saliva: Clinical Significance in Oral Diseases. Therapeutics, Pharmacology and Clinical Toxicology, 15, 139-143. 